\title{
An Integrated Structure for Supplier Selection and Configuration of Knowledge-Based Networks Using QFD, ANP, and Mixed-Integer Programming Model
}

\author{
M. Abbasi, R. Hosnavi, and B. Tabrizi \\ Department of Management and Soft Technologies, Malek-e-Ashtar University of Technology, Tehran, Iran \\ Correspondence should be addressed to B. Tabrizi; btabrizi_maut@yahoo.com
}

Received 21 June 2013; Accepted 17 September 2013

Academic Editor: Ihsan Kaya

Copyright (c) 2013 M. Abbasi et al. This is an open access article distributed under the Creative Commons Attribution License, which permits unrestricted use, distribution, and reproduction in any medium, provided the original work is properly cited.

Today's competitive world conditions and shortened product life cycles have led to the rise of attention towards new product development issue which can guarantee both growth and survival of organizations. The agility of new product development is directed by the efficiency and efficacy of knowledge management skills of an organization. A key issue in thorough success of such networks is the developed knowledge preservation amongst the members. Thus, it is important that reliable relations can be established between the members in order to promote further interactions. To do so, an integrated framework is developed in this paper to configure the new product development network so that sustainable collaborations can be maintained amongst the entities. The proposed framework consists of the network configuration in addition to the supplier selection phase. They are taken into consideration using a biobjective mathematical model in which incurred costs and suppliers' superiority determine the final configuration of the network. Finally, different numerical instances are solved to address the applicability of the proposed model.

\section{Introduction}

Over the last years, engineering and manufacturing companies have extensively concentrated on developing innovative and new products, improving their value and design for modern goods and supplies. The emergence and rise of consumer demands for novel products, shortened product life cycles, and contemporary technological developments have highlighted the demand for new and innovative products.

The aforementioned issues make the organizations burdened with significant financial and opportunity losses if the development or launch of their new product is postponed. For instance, Kurawarwala and Matsuo [1] stated that a six-eight-month delay in the launch of products such as computers and cellular phones by a computer manufacturer results in a $50-75 \%$ loss in revenue. In a more recent study, McGrath and MacMillan [2] showed that a six-month delay in the introduction of the product would decrease the project's net present value by over $\$ 2$ million, with all other parameters remaining constant. These stimuli make organizations increasingly pay attention to their new product development capabilities, increase efficiency, reduce development costs, and slash the overall cycle time.

New product development (NPD) is an iterative process of gathering, creating, and evaluating information for developing new, defect-free, and quality products. The agility of NPD is governed by the efficiency of knowledge management skills and knowledge creation cycle, which are largely and directly influenced by the organization's internal and external collaboration capability [3]. When technological and social collaborations are recognized in an organization, knowledge losses are effectively coped with in the system [4].

Knowledge loss, in context of NPD, is interpreted as the loss of knowledge, information, and experience among the entities or departments. As the efficiency of the NPD is associated with the robustness of the knowledge management system, identification, creation, and transfer of understandings and experiences through the network become critical.

It should be noted that the question of turnover or longterm absence is taken into account as a restraining factor in knowledge management [5]. Liebowitz [6] declares that if there are not a tailored knowledge retention program 
and strong leadership within a network, it suboptimizes and becomes susceptible to knowledge loss. In fact, a strong focus on retaining knowledge amongst the participating members throughout all levels of the network is needed for sustainability [7]. Hence, it is of a great importance for the network to establish its collaborations based on a sustainable framework. In other words, as the suppliers stay at the top tier in a network, their selection in terms of reliable and sustainable criteria can generalize a dependable flow down the stream and help the implicit knowledge be kept within the tiers.

Referring to the literature, there is a noticeable amount of works that have addressed the sustainable network configuration and supplier selection, in particular. However, they have just taken the problem into account with respect to the economic, environmental, and social aspects. In fact, in these papers the sustainability has been restricted by the aforementioned facets and no connection has been addressed towards knowledge preservation importance in decisions.

Xiwei et al. [8] considered the knowledge-based networks $(\mathrm{KN})$ with respect to concepts, mechanism, and components by three distinctive categories including the macro-, medium, and microlevels. The macrolevel corresponds with activities raising innovation at a national level. The medium level encompasses companies' business activities that are centered within knowledge flows, and the microlevel is associated with activities between functional areas. Theoretical research on $\mathrm{KN}$ chiefly emphasizes its characteristics and models (e.g., see $[9,10])$.

The KN can be defined just like a physical supply network which involves matching supplies to customers' demand. In other words, the most important factor in new product development knowledge-based networks (NPDKN) can be affiliated with the characteristics and potential of long-term usage of the obtained knowledge. Therefore, the network should be designed not only with respect to the overall costs, but also with regard to the possibility of close relation and trust between the suppliers and manufacturers.

For instance, Sams et al. [11] considered the sustainability paradigm by taking the key business decisions such as environment, ecology, and economy for the small retailers in the United States into account. de Carvalho and Barbieri [12] analyzed the induction process of technological innovations that reflect economic, social, and environmental concerns within the supply chain, compliant with the sustainability concept. They considered the role of central companies as innovation inductors for their supply chains. Moreover, they discussed the points of innovation, sustainability, and supply chain management. A case study was performed on a cosmetics company which depicted that sustainable innovation driven by the core company required the engagement of its suppliers to decrease the negative social and environmental impacts throughout the product's life cycle. Moreover, it was illustrated that the network could benefit from social and environmental points with respect to the innovation issue. Shymko and Diaz [13] addressed the mechanisms that trigger the sustainability of partnership model in buyersupplier relations. They investigated the role of context and actors in the dynamics of strategic alliance development and their effect on its subsequent sustainability. Their proposed model links alliance sustainability with different conditions of supply chain's internal and external environments enhanced by a case study on the evolution of Rolls-Royce and Airbus partnership in the aviation industry.

As stated earlier, supplier selection is one of the important processes in supply chain management. The importance can be more highlighted once are to be taken governmental legislations and market demands into account in the selection phase. Amindoust et al. $[14,15]$ pointed that all components of the collaborating network should have affinity with sustainability paradigm according to the growth of knowledge on sustainability issues in supply chain management. They applied data envelopments analysis to select appropriate suppliers. Amindoust et al. $[14,15]$ also indicated the importance of sustainable supplier selection with respect to the growth of knowledge in enterprises, as the key component in the sustainable supply chain management.

Govindan et al. [16] considered sustainable supply chain initiatives and addressed the problem of determining an effective model based on the triple bottom line (TBL) approach, that is, economic, environmental, and social aspects, for supplier selection operations in supply chains by presenting a fuzzy multicriteria approach. They used triangular fuzzy numbers to express linguistic values of experts' subjective preferences. Qualitative performance evaluation was first carried out by using fuzzy numbers to obtain criteria weights, and fuzzy TOPSIS was applied to rank the suppliers, afterwards. Azadnia et al. [17] explored all aspects of sustainability for supplier selection problem in an integrated assessment. They proposed an integrated approach of analytical hierarchy process (AHP) and fuzzy logic to solve the sustainable supplier selection problem. Fuzzy AHP was used to calculate the weight of sustainable criteria and sub criteria. Afterwards, fuzzy logic was applied to assess the suppliers according to the weights obtained from fuzzy AHP. Tseng and Chiu [18] introduced environmental management systems, profitability of supplier, and supplier relationship closeness as the most important factors in supplier assessment, respectively.

On the other hand, supplier selection and supply chain network configuration are associated with the supply management phase of the supply chain management, that is, the first management phase [19]. Referring to the literature, a large amount of papers can be found on the network configuration problem from which a few ones are mentioned here. For example, Amiri [20] proposed a heuristic solution approach for a multisource network configuration problem. Çakir [21] considered a multicommodity multimode distribution planning problem and applied the Benders decomposition technique to solve the mixed-integer programming model. Altiparmak et al. [22] developed a multistage multicommodity network configuration problem and applied a steady-state genetic algorithm as an efficient solution approach. Susarla and Karimi [23] integrated procurement, production, and distribution along with the effects of international tax differentials, inventory holding costs, material life cycles, and waste recovery/disposal for a multiperiod planning problem. 
As can be seen, an enriched set of research works can be found in each phase of the supply management, distinctively. However, few papers have proposed a tailored integration method, at least to the best knowledge of the authors. On the other hand, the knowledge flow preservation should be regarded as a crucial issue throughout the network whose reasons were indicated before. In other words, it is required to configure sustainable networks so that they can practice efficiently for developing new products, in particular. The simultaneous consideration of supplier selection and network configuration at the outset makes it possible to form a network that utilizes the suppliers that can help the other members during the chain practices the best. Hence, a biobjective problem is developed in this paper to select the suppliers based on a reliable background in addition to configuration at the minimum costs.

The rest of this paper is organized as follows. Problem formulation is presented in Section 2, which incorporates both the supplier selection and network configuration issues. It discusses the way in which QFD and analytic network process (ANP) are used to rank the relative importance of attributes. The accuracy and applicability of the proposed model are checked by some instances in Section 3. Finally, conclusions and future research directions are mentioned in Section 4.

\section{Problem Formulation}

In this section, the supplier selection and network configuration problems are discussed. As a general rule, the suppliers are qualified and rated in accordance with their core competencies. Likewise, the configuration of a network can be evaluated with respect to the corresponding costs of different practices of extant members. The most common costs of a given network include the annual fixed costs, that is, the cost of opening manufacturing plants, and logistics costs, that is, the cost of supply and transport of commodities from suppliers to customers, respectively.

2.1. Supplier Selection. The supplier selection process is considered here by applying quality function deployment (QFD) and ANP, respectively. The QFD is utilized in order to map the customer's attributes (CAs) into the engineering characteristics (ECs) and ANP is applied to systematically take into account the interrelationship between and within the QFD components. Furthermore, triangular fuzzy numbers (TFN) are used to deal with the vagueness and imprecision of the judgments associated with the ANP pairwise comparisons.

2.1.1. Quality Function Deployment. Mitsubishi Heavy Industries first introduced the QFD concept in 1972. Afterwards, Toyota and its suppliers developed the concept in design problems in automobile manufacturing application during 1970s. Toyota could reduce its startup and preproduction costs by $60 \%$ between 1977 and 1984 by this method. QFD is a customer-oriented tool in ranking the CAs, in regard to ECs, as it provides a conceptual map for cross-functional planning and communication [24]. Its main purpose is to decrease two types of conflict. The former is that the commodity specification does not conform to the CAs and the latter is that the final commodity is not in accordance with the ECs. The remarkable achievements of the Japanese companies by using QFD led to a speedy spread throughout the American companies in 1980s.

The QFD is usually made up of several matrices, called house of quality (HoQ), shown in Figure 1. As can be seen, the matrix is made up of many parts that construct the house parts. The roof of the house refers to the interaction of ECs (HOWs). In fact, ECs stand for different ways and alternatives in which each one can distinctively meet the criteria. The matrix embedded in the left of the HoQ encompasses the CAs (WHATs). In fact, CAs represent the chief criteria in achieving the NPDKN goal. Moreover, the market evaluation (i.e., customer perception and its target value) is placed on the right-hand side. Finally, the technical competitive benchmark and EC target value are located at the bottom.

A large extent of consideration was carried out through the literature in order to extract the appropriate attributes (e.g., [25-28]). As there was not found a comprehensive set of criteria to represent the supplier selection in an NPDKN environment, the following attributes are determined with respect to similar research and the experts' opinions:

(1) delivery time: the required time to deliver the orders and its stability,

(2) equity acceptance: the attitude towards playing equally important roles in managing the network,

(3) laws, regulations, and standards abiding by: obeying the laws and considering standards, patents, and, and so forth, to develop new products,

(4) health and security: the quality of the commodities,

(5) flexibility: the possibility and ability to supply and develop new products,

(6) loyalty: attention to morals and professional ethics to do the best,

(7) willingness to long-term participation: the inclination to establish long-run collaborations,

(8) accessibility and customers' support: the availability once needed and the reputation amongst the customers.

The previous attributes can be mainly divided into two groups including the quality criteria of the suppliers and abiding by thorough achievement of the entire network. Moreover, the ECs are given as follows:

(1) cooperation with suppliers: the cooperation strategies,

(2) optimized work and knowledge flow: the maturity of work and knowledge flows,

(3) use of effective management information systems: attention to the information systems as a vital affair,

(4) integration of new technologies: orientation towards the application and integration of new and high technologies, 


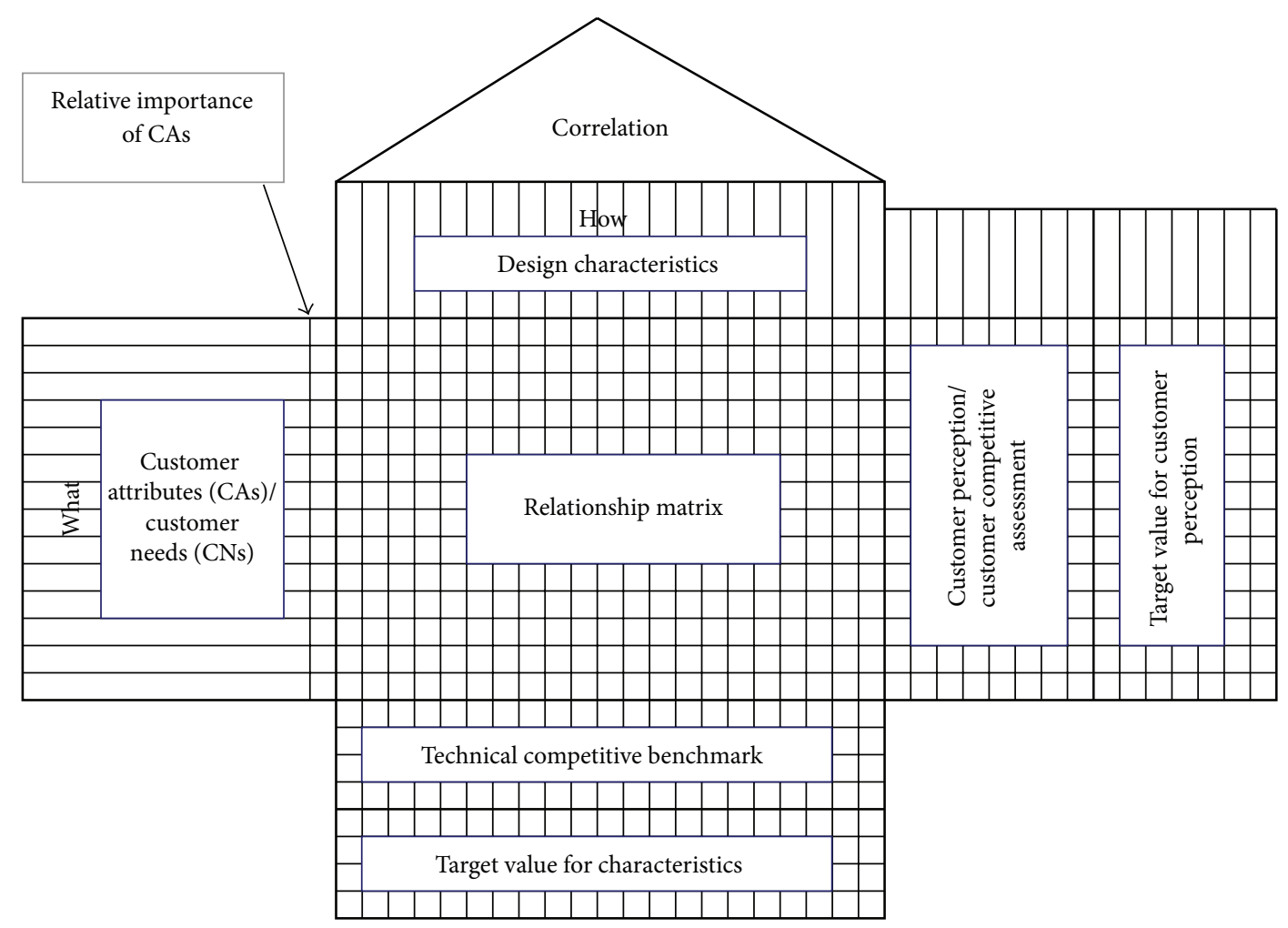

FIgUre 1: The HoQ.

(5) quality system certification: the necessity of documentation and processes tracking,

(6) financial and economic stability: the financial capability in the case of crisis, in particular,

(7) innovation and novelty capacity: the liability and potential of innovation,

(8) past records and performance: the reputation and attitudes amongst the people.

2.1.2. Analytic Network Process. Saaty [29] developed ANP as an extension of AHP based on the network construction. The ANP relies on a two-stage modeling including the development of a network diagram and priorities determination of the elements. The strength point of this modeling method goes back to the interaction incorporation of the components.

The given network is then translated into a supermatrix representing the dependence level amongst the constructing components. The priority vectors are calculated by pairwise comparisons. The super matrix is then raised to limiting powers to determine the overall priorities of the elements and the influence of each element on any other elements, as well. In other words, the implementation steps of ANP can be briefly explained as follows:

(1) developing a (nonlinear) network system with regard to the decision-maker's or expert's opinions; however, it should be noted that the process can be followed by a group of decision-makers/experts, instead,
(2) establishing an original unweighted super matrix. In this phase, the local weight matrix is obtained in which the influence amount of a given cluster is determined over the other ones,

(3) identifying the weight matrix of the network clusters which is performed by the pairwise comparisons,

(4) developing the weighted super matrix which can be obtained by the multiplication of the cluster matrices and un-weighted super matrix,

(5) calculating the final weighted super matrix which shows the final rank (score) of the elements of each cluster.

Since a QFD analysis process can be regarded as a decision problem, it can be formulated as an ANP model. The issue can be illustrated in Figure 2 in which the goal is related to the appropriate collaboration of the network members with respect to the selected suppliers. Likewise, criteria and alternatives pertain to the CAs and ECs, respectively. Therefore, the QFD framework can be easily represented by a network diagram. The bottom line in application of ANP lies in the way of establishment of the decision network which is dealt here with QFD. In other words, the QFD specifies the clusters (i.e., the criteria and alternatives) of ANP such that the appropriate weights of each element can be obtained.

2.1.3. Triangle Fuzzy Number. Since most of the required data of QFD are supplied from customers or decision makers and human expressions are not usually precise, linguistic terms 


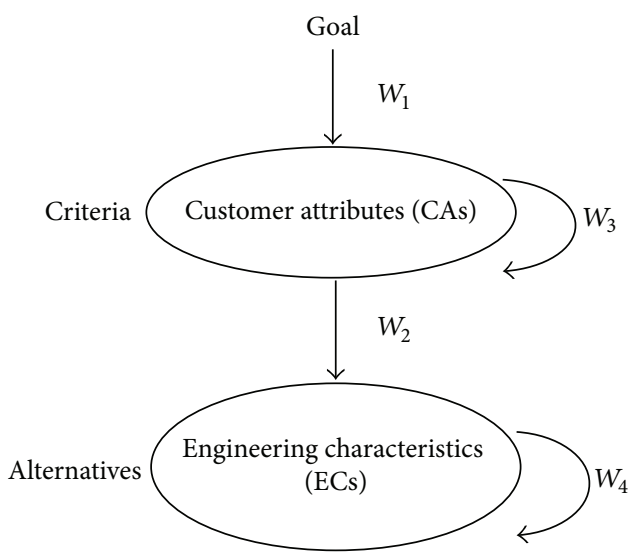

FIGURE 2: Network diagram illustration of a QFD.

are used as an appropriate way to deal with the corresponding vagueness. For example, the human expressions may be stated in terms of linguistic variables such as "extremely important," "fairly important," and so forth. However, they are accompanied by imprecision which can be easily treated by fuzzy set theory introduced by [30]. Virtually, linguistic values can be represented by different fuzzy-shaped numbers (e.g., triangular or trapezoidal).

It is here assumed that linguistic values can be appropriately represented by TFNs, commonly used in the literature. A TFN $\widetilde{N}$ can be defined as a triplet $(l, m, u)$, shown by Figure 3 . The corresponding membership function $\mu_{\widetilde{N}}(x)$ is obtained by (1), where $l, m$, and $u$ are real numbers and $l \leq m \leq u$ :

$$
\mu_{\widetilde{N}}(x)= \begin{cases}0 & x<l, \\ \frac{(x-l)}{(m-l)} & l \leq x \leq m \\ \frac{(u-x)}{(u-m)} & m \leq x \leq u \\ 0 & x>u .\end{cases}
$$

Table 1 shows the linguistic terms used here to express the attributes. Once the judgments are gathered in terms of the linguistic variables, the application of an efficient fuzzy aggregation method to consider the interval by which the TFN is defined is needed. Any fuzzy aggregation method requires a defuzzification method representing the selection

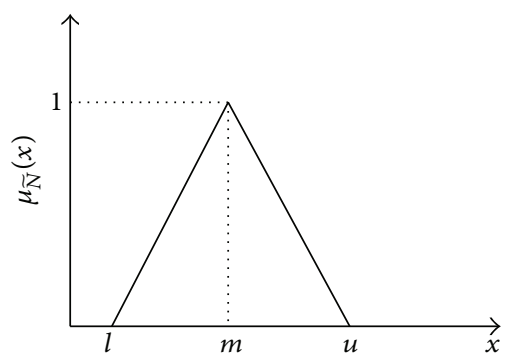

FIGURE 3: A symmetric TFN.

TABLE 1: Linguistic variables used to express the relative importance of the attributes.

\begin{tabular}{lc}
\hline Linguistic term & Fuzzy numbers \\
\hline Very low & $(0,0,0.1)$ \\
Low & $(0,0.1,0.3)$ \\
Medium low & $(0.1,0.3,0.5)$ \\
Medium & $(0.3,0.5,0.7)$ \\
Medium high & $(0.5,0.7,0.9)$ \\
High & $(0.7,0.9,1)$ \\
Very high & $(0.9,1,1)$ \\
\hline
\end{tabular}

of an explicit crisp element based on the fuzzy set. There are many different defuzzification methods; however, a good defuzzification method should address the fuzzy number with respect to its shape, spread, height, and relative location on the $x$-axis. For example, Centroid (center-of-gravity) method does not distinguish between two fuzzy numbers with the same crisp value despite different shapes [31]. The authors chose Liu and Wang's defuzzification method [32] for fuzzy for this purpose which benefits from a rather simple structure. Their proposed approach is based on both the area method and the radius of gyration (ROG) points. The aggregation procedure had been considered for trapezoidal fuzzy numbers and was then modified by Liu et al. [33] for TFNs. The area $S(\widetilde{A})$, between the ROG point and the original point of a fuzzy number, can be determined as follows:

$$
S(\widetilde{A})=r_{x} \cdot r_{y}
$$

As the area $S(\widetilde{A})$ is larger, the fuzzy number is larger. Regarding Liu and Wang [32] for a TFN, $S(\widetilde{A})$ can be calculated by (3), in which $r_{x}$ and $r_{y}$ are obtained through (4) and (5), respectively:

$$
\begin{gathered}
S(\widetilde{A})=\sqrt{\frac{(m-l)^{3} / 4+(m-1) l^{2} / 2+2(m-l)^{2} l / 3+(u-m)^{3} / 12+(u-m) m^{2} / 2+(u-m)^{2} m / 3}{3(u-l)},} \\
r_{x}=\sqrt{\frac{1}{6}}, \\
r_{y}=\sqrt{\frac{(m-l)^{3} / 4+(m-1) l^{2} / 2+2(m-l)^{2} l / 3+(u-m)^{3} / 12+(u-m) m^{2} / 2+(u-m)^{2} m / 3}{(u-l) / 2}} .
\end{gathered}
$$


The total performance of each supplier can be obtained with regard to its capabilities in each attribute and the relative importance of the given attribute when they are evaluated from the integrated QFD-ANP approach. Consider that $\mathrm{RI}_{i}$ and $\mathrm{ECC}_{\text {sic }}$ represent the relative importance of the $i$ th attribute and engineering characteristics capability of supplier $s$ with respect to the $i$ th attribute for commodity $c$, respectively. Moreover, take $x_{s c p}$ as a binary variable which equals 1 if supplier $s$ is selected to supply $c$ th commodity for plant $p$ and 0 , otherwise. Thus, the objective function of the supplier selection utility can be written as follows:

$$
\operatorname{Max} f_{1}=\sum_{i=1}^{I} \sum_{s=1}^{S} \sum_{c=1}^{C} \sum_{p=1}^{P} \mathrm{RI}_{i} \cdot \mathrm{ECC}_{s i c} \cdot x_{s c p} .
$$

2.2. Network Optimization. In this section, the network configuration problem of NPS is taken into account. Different commodities are considered here to underlie the suitable background of developing new products. It is assumed that the network consists of suppliers, plants, and distribution centers. It is also assumed that manufacturing facilities and distributors can be supplied by multisources. The proposed mathematical model tries to minimize the annual fixed cost, associated with open or close decisions of plants, and logistics costs, including processing and transportation costs.

Let us suppose $y_{p}$ as a binary variable that takes 1 if plant $p$ is open and 0 , otherwise. $\mathrm{FC}_{p}^{1}$ shows the annual fixed cost of opening plant $p . \mathrm{FC}_{s}^{2}$ also represents the fixed cost of selection of supplier $s$ which can be interpreted in terms of the communications, correspondence, and contract costs. Furthermore, $q_{s c p}^{1}$ and $q_{p c d}^{2}$ stand for the quantity of commodity $c$ transported from supplier $s$ to plant $p$ and from plant $p$ to distributor $d$, respectively. Hence, the objective function can be written by (7) in which $l_{s c p}^{1}$ and $l_{p c d}^{2}$ represent the logistics costs for processing and transporting commodity $c$ from supplier $s$ to plant $p$ and from plant $p$ to distributor $d$, respectively,

$$
\begin{aligned}
\operatorname{Min} f_{2}= & \sum_{p=1}^{P} \mathrm{FC}_{p}^{1} y_{p}+\sum_{s=1}^{S} \sum_{c=1}^{C} \sum_{p=1}^{P} \mathrm{FC}_{s}^{2} x_{s c p} \\
& +\sum_{s=1}^{S} \sum_{c=1}^{C} \sum_{p=1}^{P} q_{s c p}^{1} l_{s c p}^{1}+\sum_{p=1}^{P} \sum_{c=1}^{C} \sum_{d=1}^{D} q_{p c d}^{2} l_{p c d}^{2} .
\end{aligned}
$$

The constraints of the given mathematical model are written through as follows:

$$
\begin{gathered}
\sum_{p=1}^{P} q_{s c p}^{1} \leq \mathrm{Ca}_{s c} ; \quad \forall s, c, \\
\sum_{p=1}^{P} x_{s c p} \leq S ; \quad \forall s, c, \\
x_{s c p} \leq q_{s c p}^{1} ; \quad \forall s, c, p,
\end{gathered}
$$

TABLE 2: The normalized weights of each attribute.

\begin{tabular}{lc}
\hline Attributes & Normal weights \\
\hline Delivery time & 0.11 \\
Equity acceptance & 0.04 \\
Abiding by Laws, regulations, and standards & 0.14 \\
Health and security & 0.08 \\
Flexibility & 0.16 \\
Loyalty & 0.23 \\
Willingness to long-term participation & 0.18 \\
Accessibility and customers' support & 0.06 \\
\hline
\end{tabular}

TABLE 3: Data generation method.

\begin{tabular}{lc}
\hline Parameter & Random distribution function \\
\hline $\mathrm{FC}_{p}^{1}$ & $\sim U[75000,100000]$ \\
$\mathrm{FC}_{s}^{2}$ & $\sim U[100000,150000]$ \\
$l_{s c p}^{1}$ & $\sim U[150,300]$ \\
$l_{p c d}^{2}$ & $\sim U[100,150]$ \\
$\mathrm{Ca}_{s c}$ & $\sim U[120,160]$ \\
$\mathrm{Ca}_{p c}$ & $\sim U[80,120]$ \\
$\mathrm{De}_{d c}$ & $\sim U[30,60]$ \\
\hline
\end{tabular}

$$
\begin{gathered}
\sum_{d=1}^{D} q_{p c d}^{2} \leq \sum_{s=1}^{S} q_{s c p}^{1} ; \quad \forall c, p, \\
\sum_{d=1}^{D} q_{p c d}^{2} \leq \mathrm{Ca}_{p c} ; \quad \forall c, p, \\
\sum_{p=1}^{P} q_{p c d}^{2} \geq \mathrm{De}_{d c} ; \quad \forall c, d, \\
q_{s c p}^{1}, q_{p c d}^{2} \geq 0 ; \quad y_{p}, x_{s c p} \in\{0,1\} .
\end{gathered}
$$

Equation (8) shows that the transported volume of commodity $c$ from supplier $s$ cannot exceed the corresponding supplier's capacity. Equation (9) states that the total number of selected suppliers should not exceed the predefined upper bound. Equation (10) guarantees that no commodity supply is met unless the relevant supplier is selected. Equation (11) determines that the amount of commodity transported from a plant to the customers cannot exceed the entered flow of the commodity to the given plant. Equation (12) shows that the volume of transported commodities to the customers cannot surpass the plants' operational capacity. Moreover, (13) points to the necessity of satisfying the customers' demands. Finally, the variables are defined by (14).

As the mathematical model consists of two objective functions, the application of an efficient method to deal with the given problem is needed. The global criterion method is utilized here which is based on the dimensions elimination of the problem solution space. It should be noted that the model has been developed so that the sum square of deviations 
TABLE 4: The results obtained from different instances.

\begin{tabular}{|c|c|c|c|c|c|c|c|c|}
\hline \multicolumn{3}{|c|}{ Small size } & \multicolumn{3}{|c|}{ Medium size } & \multicolumn{3}{|c|}{ Large size } \\
\hline$s \times c \times p \times d$ & $\begin{array}{c}\text { Objective } \\
\text { function }(\$)\end{array}$ & $\begin{array}{c}\text { Elapsed } \\
\text { time* }^{*}(\text { Sec. })\end{array}$ & $s \times c \times p \times d$ & $\begin{array}{c}\text { Objective } \\
\text { function (\$) }\end{array}$ & $\begin{array}{c}\text { Elapsed } \\
\text { time }^{*}(\text { Sec. })\end{array}$ & $s \times c \times p \times d$ & $\begin{array}{c}\text { Objective } \\
\text { function }(\$)\end{array}$ & $\begin{array}{c}\text { Elapsed } \\
\text { time* }^{*}(\text { Sec. })\end{array}$ \\
\hline $2 \times 2 \times 5 \times 8$ & 0.14 & 7 & $3 \times 3 \times 8 \times 15$ & 0.18 & 108 & $5 \times 3 \times 12 \times 25$ & 0.15 & 1638 \\
\hline $3 \times 2 \times 6 \times 12$ & 0.09 & 18 & $3 \times 3 \times 10 \times 18$ & 0.12 & 195 & $6 \times 3 \times 15 \times 30$ & 0.12 & 3104 \\
\hline $2 \times 3 \times 5 \times 10$ & 0.12 & 44 & $4 \times 3 \times 10 \times 20$ & 0.16 & 355 & $6 \times 4 \times 15 \times 30$ & 0.11 & 5765 \\
\hline $2 \times 3 \times 6 \times 12$ & 0.23 & 63 & $4 \times 3 \times 12 \times 25$ & 0.23 & 490 & $6 \times 5 \times 18 \times 40$ & 0.13 & 8442 \\
\hline $3 \times 3 \times 6 \times 12$ & 0.17 & 79 & $5 \times 2 \times 12 \times 25$ & 0.17 & 876 & $7 \times 4 \times 20 \times 50$ & 0.18 & 14769 \\
\hline
\end{tabular}

${ }^{*}$ shows the aggregate solution time calculated for each of the objective functions independently in addition to the final model solution elapsed time, that is, model (15).

can be minimized. The formulation can hence be written as follows:

$$
\begin{aligned}
& \text { Min } f=\left[\frac{f_{2}-f_{2}^{*}}{f_{2}^{*}}\right]^{2}-\left[\frac{f_{1}-f_{1}^{*}}{f_{1}^{*}}\right]^{2} \\
& \text { S.t. }(8)-(14) .
\end{aligned}
$$

\section{Experimental Results}

In this section, some different sized instances are taken into consideration to check the formulation performance and its accuracy. First, the attributes are ranked by the integrated framework of QFD-ANP from which the relative weights are shown in Table 2. In order to obtain the final weights of the attributes, Super Decision software was utilized such that calculations effort is reduced. It should be noted that each of the pairwise comparisons was carried out in a way that the corresponding consistency ratio was acceptable. However, the application steps of ANP could be implemented regarding mathematical calculations. The rest of the required data are generated by appropriate random distributions, shown in Table 3. However, the upper bound of selected suppliers is determined with respect to problem size. The calculations are run by the ILOG CPLEX 10.1 optimization software under a PC with characteristics of Intel(R), Pentium(R) 4, CPU $3.20 \mathrm{GHz}$, and $2 \mathrm{~GB}$ of RAM.

Table 4 shows the results obtained from different instances solved by the global criterion method, in which they are divided into small, medium, and large sizes. It should be noted that the global criterion method requires the given problem to be solved with respect to the number of objective functions. Hence, for each instance, the problem is solved regarding each objective function, distinctively. Afterwards, the optimal values of the objective functions are used to write model (15). As can be seen, the results are compared with respect to the CPU time and the objective function values. The objective function values are all between 0 and 1 , as each objective has been normalized. On the other hand, the required elapsed time has been raised to a remarkable extent for the larger sizes, in particular. In fact, the complexity rise of the given problem requires it to spend more computational effort to reach the final solution. However, the efficient solutions of all instances have been obtained here which shows the applicability of the proposed method.

\section{Conclusions}

The severe competition amongst the companies and manufacturing firms to get more market shares and shortened products life cycle highlights the essence of NPD. Hence, the configuration problem of NPD networks was addressed in this paper. The proposed model consisted of an integrated framework for supply management including simultaneous supplier selection and network design. The QFD and ANP were applied to rank the relative importance of the key attributes in selection of the suppliers. Moreover, fuzzy set theory was used to deal with the intrinsic imprecision of the linguistic judgments. Afterwards, a mixed-integer programming mathematical model was formulated to consider the KN configuration. Finally, different sized instances were taken into consideration by the global criterion method to check the efficiency and applicability of the model.

The proposed model benefits from simultaneous decision making on the network planning and suppliers selection. This issue can lead to an enhanced set which can select the suppliers with respect to the given costs, in addition to the network reliability. However, the model may be questioned with regard to the real-world uncertainties. Therefore, as the future research interests, the uncertainty can be generalized to the given parameters. Another future research direction is to apply heuristic solution methods so that the solution time can be decreased to a remarkable extent.

\section{References}

[1] A. A. Kurawarwala and H. Matsuo, "Forecasting and inventory management of short life-cycle products," Operations Research, vol. 44, no. 1, pp. 131-150, 1996.

[2] R. G. McGrath and I. C. MacMillan, "How to rethink your business during uncertainty," MIT Sloan Management Review, vol. 50, no. 3, pp. 25-30, 2009.

[3] R. Shankar, N. Mittal, S. Rabinowitz, A. Baveja, and S. Acharia, "A collaborative framework to minimise knowledge loss in new product development," International Journal of Production Research, vol. 51, pp. 2049-2059, 2013.

[4] P. M. Norman, "Knowledge acquisition, knowledge loss, and satisfaction in high technology alliances," Journal of Business Research, vol. 57, no. 6, pp. 610-619, 2004.

[5] S. Durst and S. Wilhelm, "Knowledge management in practice: insights into a medium-sized enterprise's exposure to knowledge loss," Prometheus, vol. 29, no. 1, pp. 23-38, 2011. 
[6] J. Liebowitz, "What practitioners need to know," KM World, vol. 20, no. 2, pp. 12-13, 2011.

[7] P. Chinowsky and P. Carrillo, "Knowledge management to learning organization connection," Journal of Management in Engineering, vol. 23, no. 3, pp. 122-130, 2007.

[8] W. Xiwei, M. Stolein, and W. Kan, "Designing knowledge chain networks in China-a proposal for a risk management system using linguistic decision making," Technological Forecasting and Social Change, vol. 77, no. 6, pp. 902-915, 2010.

[9] I. Nonaka and N. Konno, "The concept of "Ba": building a foundation for knowledge creation," California Management Review, no. 3, pp. 40-54, 1998.

[10] T. Powell, "The knowledge value chain (KVC): how to fix it when it breaks," in Proceedings of the 22nd National Online Meeting, pp. 301-312, New York, NY, USA, May 2001.

[11] D. Sams, E. Scarboro, J. Parker, and I. Mayoylov, "Sustainability paradigm: perspective of the small retailers," WIT Transactions on Ecology and the Environment, vol. 173, pp. 355-366, 2013.

[12] A. P. de Carvalho and J. C. Barbieri, "Innovation and sustainability in the supply chain of a cosmetics company: a case study," Journal of Technology Management and Innovation, vol. 7, no. 2, pp. 144-156, 2012.

[13] Y. Shymko and A. Diaz, "A resource dependence, social network and contingency model of sustainability in supply chain alliances," International Journal of Business Excellence, vol. 5, no. 5, pp. 502-520, 2012.

[14] A. Amindoust, S. Ahmed, and A. Saghafinia, "Supplier performance measurement of palm oil industries from a sustainable point of view in malaysia," BioTechnology, vol. 6, no. 6, pp. 155158, 2012.

[15] A. Amindoust, S. Ahmed, A. Saghafinia, and A. Bahreininejad, "Sustainable supplier selection: a ranking model based on fuzzy inference system," Applied Soft Computing Journal, vol. 12, no. 6, pp. 1668-1677, 2012.

[16] K. Govindan, R. Khodaverdi, and A. Jafarian, "A fuzzy multi criteria approach for measuring sustainability performance of a supplier based on triple bottom line approach," Journal of Cleaner Production, vol. 47, pp. 345-354, 2013.

[17] A. H. Azadnia, P. Ghadimi, M. Z. M. Saman, K. Y. Wong, and C. Heavey, "An integrated approach for sustainable supplier selection using fuzzy logic and fuzzy AHP," Applied Mechanics and Materials, vol. 315, pp. 206-210, 2013.

[18] M.-L. Tseng and A. S. F. Chiu, "Evaluating firm's green supply chain management in linguistic preferences," Journal of Cleaner Production, vol. 40, pp. 22-31, 2013.

[19] C. S. Tang, "Perspectives in supply chain risk management," International Journal of Production Economics, vol. 103, no. 2, pp. 451-488, 2006.

[20] A. Amiri, "Designing a distribution network in a supply chain system: formulation and efficient solution procedure," European Journal of Operational Research, vol. 171, no. 2, pp. 567-576, 2006.

[21] O. Çakir, "Benders decomposition applied to multi-commodity, multi-mode distribution planning," Expert Systems with Applications, vol. 36, no. 4, pp. 8212-8217, 2009.

[22] F. Altiparmak, M. Gen, L. Lin, and I. Karaoglan, "A steadystate genetic algorithm for multi-product supply chain network design," Computers and Industrial Engineering, vol. 56, no. 2, pp. 521-537, 2009.

[23] N. Susarla and I. A. Karimi, "Integrated supply chain planning for multinational pharmaceutical enterprises," Computers and Chemical Engineering, vol. 42, pp. 168-177, 2012.
[24] L.-H. Chen and M.-C. Weng, "An evaluation approach to engineering design in QFD processes using fuzzy goal programming models," European Journal of Operational Research, vol. 172, no. 1, pp. 230-248, 2006.

[25] N. D. du Preez and L. Louw, "A framework for managing the innovation process," in Proceedings of the Portland International Center for Management of Engineering and Technology, Technology Management for a Sustainable Economy (PICMET '08), pp. 546-558, Cape Town, South Africa, July 2008.

[26] G. Büyüközkan and Ç. Berkol, "Designing a sustainable supply chain using an integrated analytic network process and goal programming approach in quality function deployment," Expert Systems with Applications, vol. 38, no. 11, pp. 13731-13748, 2011.

[27] M. A. Farshchi and M. Brown, "Social networks and knowledge creation in the built environment: a case study," Structural Survey, vol. 29, no. 3, pp. 221-243, 2011.

[28] L. Shen, Laya Olfat, K. Govindan, R. Khodaverdi, and A. Diabat, "A fuzzy multi criteria approach for evaluating green supplier's performance in green supply chain with linguistic preferences," Resources, Conservation and Recycling, vol. 74, pp. 170-179, 2013.

[29] T. L. Saaty, The AnaLytic Network Process. s.l, Expert Choice, RWS Publications, 1996.

[30] L. A. Zadeh, "Fuzzy sets," Information and Control, vol. 8, no. 3, pp. 338-353, 1965.

[31] B. Chang, C.-W. Chang, and C.-H. Wu, "Fuzzy DEMATEL method for developing supplier selection criteria," Expert Systems with Applications, vol. 38, no. 3, pp. 1850-1858, 2011.

[32] H.-T. Liu and C.-H. Wang, "An advanced quality function deployment model using fuzzy analytic network process," Applied Mathematical Modelling, vol. 34, no. 11, pp. 3333-3351, 2010.

[33] Z. Liu, S. Guo, L. V. Snyder, A. Lim, and P. Peng, "A p-robust capacitated network design model with facility disruptions," in Advanced Manufacturing and Sustainable Logistics, vol. 46 of Lecture Notes in Business Information Processing, pp. 269-280, 2010. 

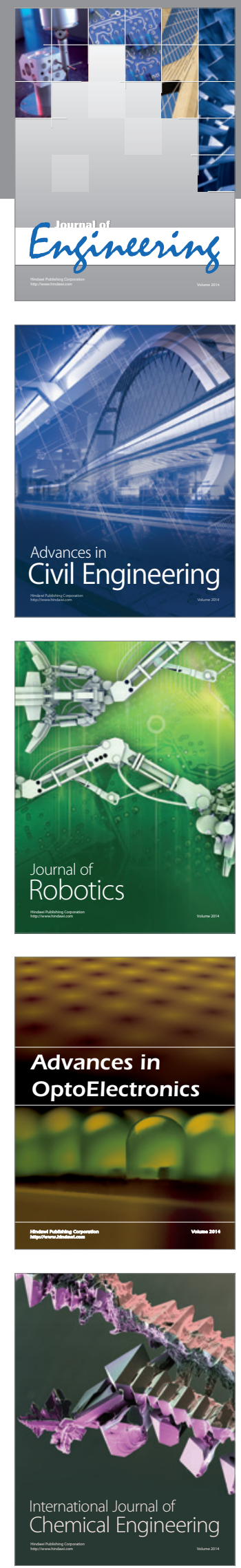

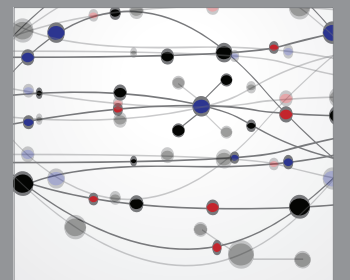

The Scientific World Journal
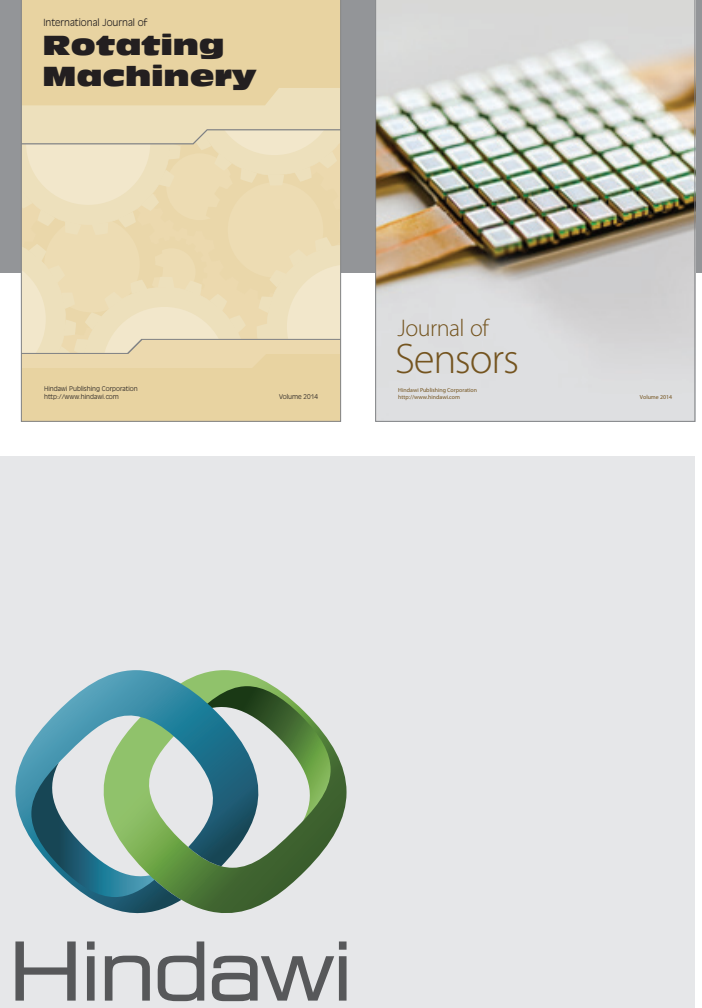

Submit your manuscripts at http://www.hindawi.com
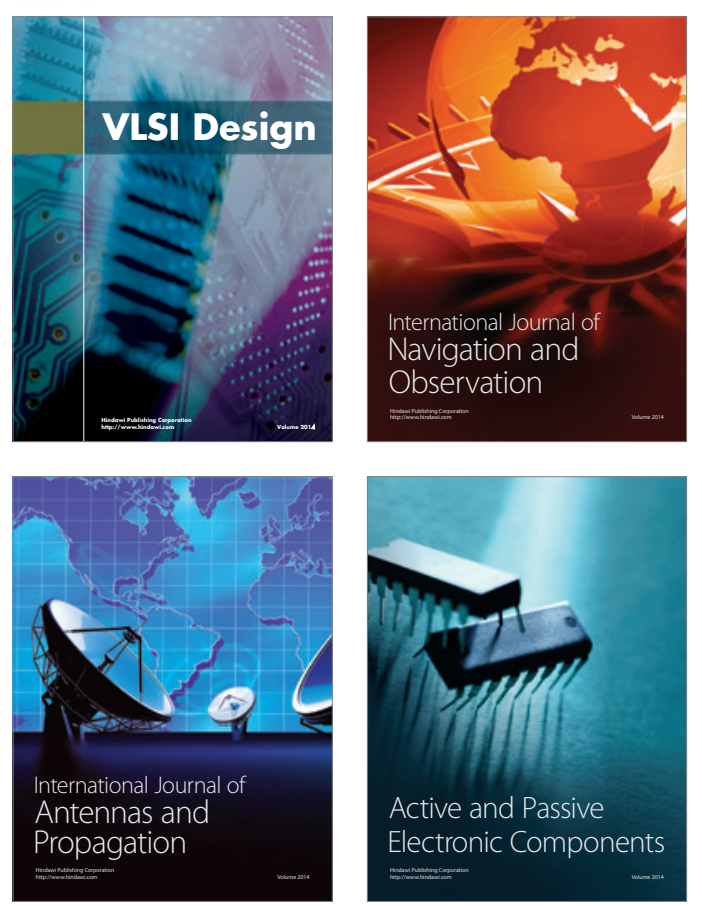
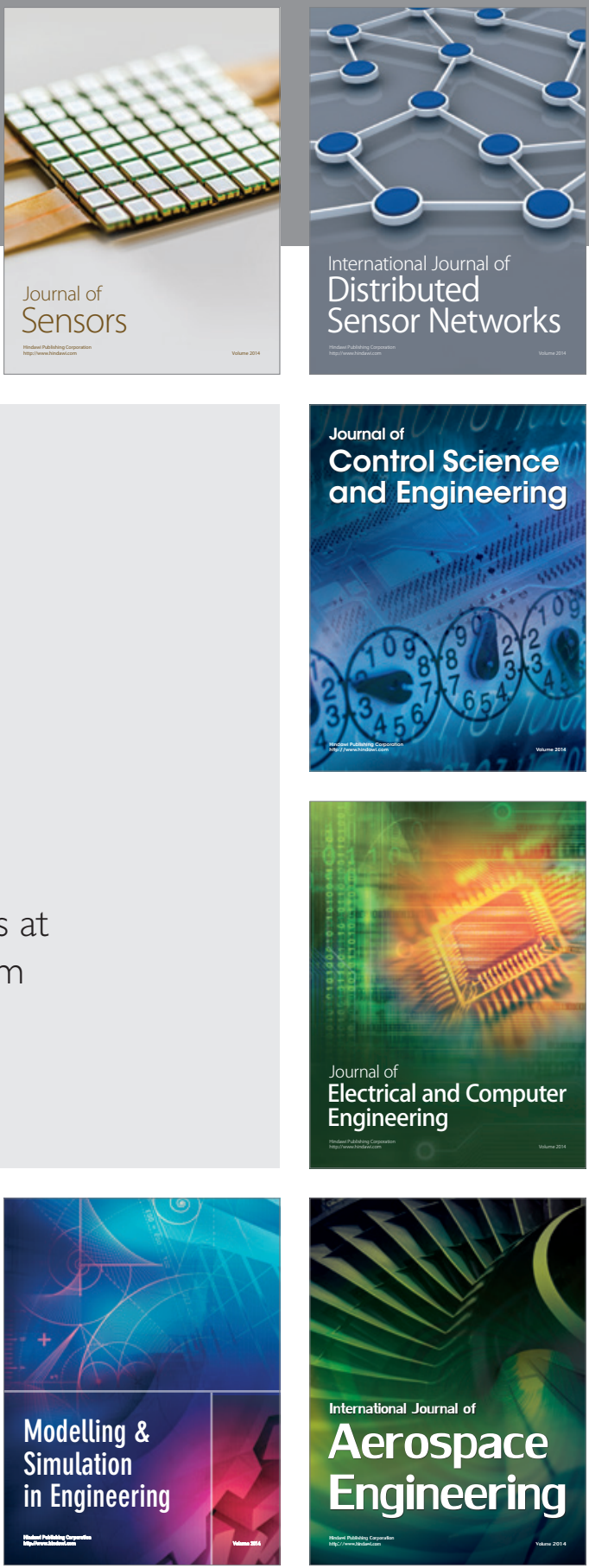

Journal of

Control Science

and Engineering
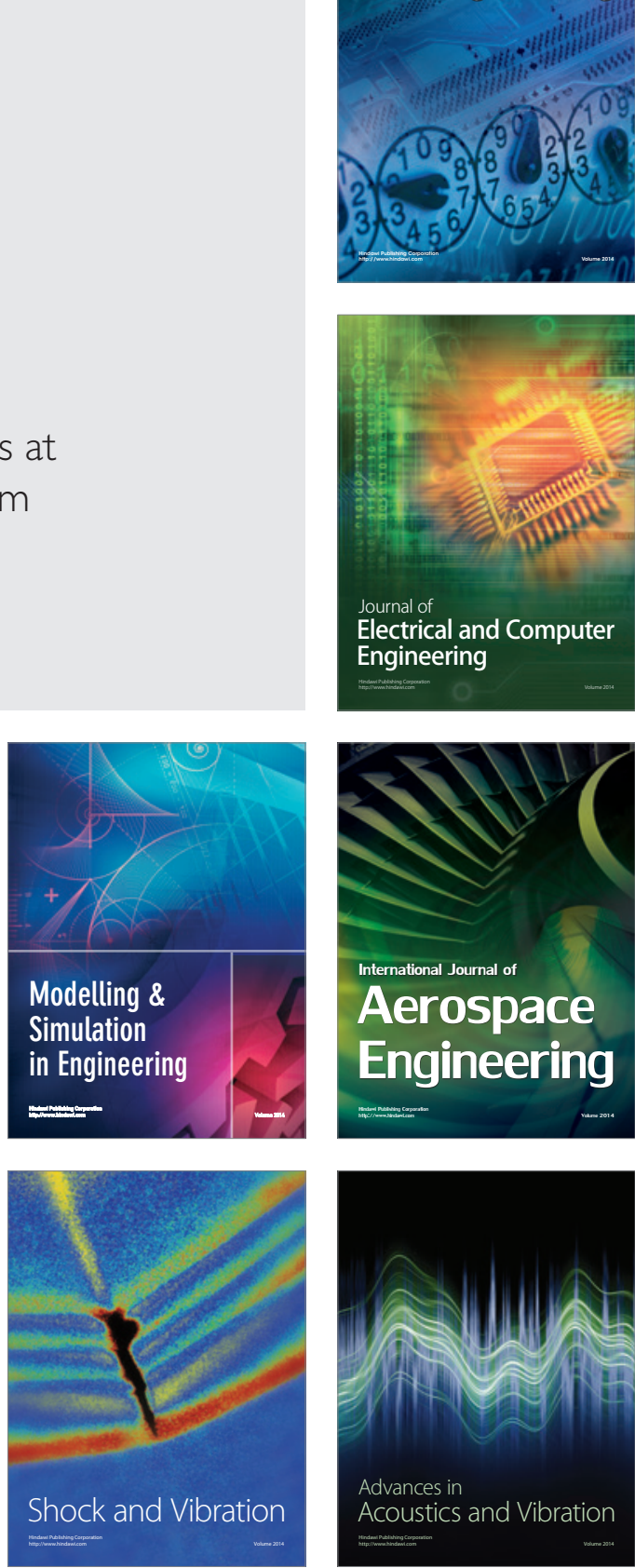